\title{
Effect of High Fructose Diet during Pregnancy on Progeny Metabolic Syndrome
}

\author{
XIAO Zi-yi1, HAN Lei² , HE Juan², ZHAO Ting², WU Ruo-nan²
}

\author{
${ }^{1}$ Public Health College of Qingdao University, Qingdao of Shandong Province, Qingdao 266021, China \\ ${ }^{2}$ Affiliated Hospital of Qingdao University, Qingdao of Shandong Province, Qingdao 266021, China
}

\begin{abstract}
Objective: To investigate the effect of high fructose diet during pregnancy on insulin resistance in the offspring. Methods: normal diet Wistar adult male rats and female rats were mated and pregnant rats were randomly divided into five groups: normal control group, normal dose group, high dose of fructose fructose group, high dose group and high dose of sucrose fructose group were given daily during pregnancy, $2 \mathrm{~mL}$ of distilled water, $2 \mathrm{~g} / \mathrm{kg}$ $18 \mathrm{~g} / \mathrm{kg}, 6 \mathrm{~g} / \mathrm{kg}, 18 \mathrm{~g} / \mathrm{kg}$ and the fructose solution of sucrose solution by gavage, continuous intervention until delivery, delivery of rats fed with basic diet, and the rats weighing body weight, fasting blood glucose. The OGTT test was conducted at the end of the eighth week. After the next day of fasting 12h, the rats were killed, anesthesia, blood extraction, liver HE staining, serum insulin level, and insulin resistance index were measured. Results: during continuous intervention, birth weight childbirth offspring rats; OGTT test: at 30min, high dose group, high dose of fructose fructose group and high dose glucose sucrose group were significantly higher than normal control group and normal group $(\mathrm{P}<0.05)$, fructose dose at $120 \mathrm{~min}$, high dose group, high dose of fructose fructose group and high dose glucose sucrose group were significantly higher than normal control group $(\mathrm{P}<0.05)$. The serum insulin and insulin resistance index of high dose fructose group, super high fructose group and super high sucrose group were significantly higher than those of normal control group and normal dose fructose group $(\mathrm{P}<0.05)$. The liver slices of high fructose group and super high fructose group showed fat accumulation. Conclusion: high fructose diet during pregnancy could increase the risk of progeny metabolic syndrome.
\end{abstract}

Keywords: Fructose; Fetal; IR ; Blood Glucose

\section{Introduction}

With the rapid development of society, people's living standard is increasing. Therefore, great changes have taken place in our country residents' dietary pattern: People's dietary patterns from a plant-based diet for Japanese dietary patterns gradually changed to animal food based dietary patterns in developed countries, it is the prevalence of chronic diseases increased: especially obesity and diabetes non communicable chronic metabolic the prevalence of the disease compared with 2002 increased. Studies have shown that ${ }^{[1]}$, the intrauterine environment of pregnant women has a vital link with the near and medium - and long - term growth and development of the offspring. A large number of epidemiological studies have confirmed that the abnormal energy metabolism in the uterus has a positive correlation with the risk of long term hypertension in the progeny of ${ }^{[2]}$.

Fructose is a monosaccharide, and natural fructose is widely found in fruit and honey. The sweetness of fructose is 1.3 times that of sucrose and can be absorbed directly. As fructose compared with sucrose, the advantage is obvious, so fructose is widely used in food processing and gradually replace sucrose. The way of fructose intake in diet can be divided into two categories: the first kind is soft drink and food with fructose as sweetener, and the other is fructose rich fruit. In China, the main intake of fructose in pregnancy is the latter.

\begin{abstract}
Materials and methods experimental animal

30 Wistar female rats (grade SPF) of 10 weeks old were selected, 15 male rats (SPF grade) and $250+20 \mathrm{~g}$ in weight. Purchased from Ji'nan Peng Yue experimental animal breeding Co., Ltd., production certificate number SCXK (Lu) 20140003.
\end{abstract}

\section{Reagents and instruments}

D- fructose: the United States Amresco, the purity of $>99 \%$; sucrose: Amresco, purity of more than 99\%; electronic balance: Ohaus instrument (Shanghai) Co. Ltd.; low temperature centrifuge: the United States Sigma company. ELx808 enzyme labeling instrument, American BioYek company; insulin ELISA detection kit, Wuhan gene beauty Biotechnology Co., Ltd.

\section{Method \\ Animal grouping and feeding \\ When 30 female rats were fed $1 \mathrm{~W}$ adaptively, they were found to be pregnant on the basis of male and female 2:1 cage. The next day, the sperm was found to be pregnant for 0 days. It was labeled as pregnant}

This article is published under the terms of the Creative Commons Attribution License 4.0

Author(s) retain the copyright of this article. Publication rights with Alkhaer Publications.

Published at: http://www.ijsciences.com/pub/issue/2018-03/

DOI: 10.18483/ijSci.1579; Online ISSN: 2305-3925; Print ISSN: 2410-4477 
female rats. After two weeks, the female rats were not given up. Pregnant rats were randomly divided into five groups, 6 in each group, and $3 \mathrm{~W}$ was continuously intervened by gavage every day. The normal control group (BC group) ( $2 \mathrm{~mL}$ distilled water), normal dose fructose group (NF group) ( $2 \mathrm{~g} / \mathrm{kg}$ fructose solution), high dose fructose group (HF group) $(6 \mathrm{~g} / \mathrm{kg}$, ultra high fructose solution) dose fructose group (SHF group) $(18 \mathrm{~g} / \mathrm{kg}$, high dose of fructose solution) sucrose group (SHS group $(18 \mathrm{~g} / \mathrm{kg})$ sucrose solution). Each delivery of pups were randomly selected 9 only basal diet.

\section{Observation index and detection method}

(1) measurement of weight: the electronic weighing method provided by the animal experiment center is called the general weighing method. (2) OGTT test: fasting $12 \mathrm{~h}$, the next morning the needle tail take around capillary blood, measuring blood glucose meter. Then the $50 \%$ glucose solution was administered to the stomach $(2 \mathrm{~g} / \mathrm{kg})$ and measured in a fast blood glucose meter for one hour and two hours. (3) determination of FINS: fasting $12 \mathrm{~h}$, taking blood from the abdominal aorta on the following day, separating serum, and using ELISA for detection in accordance with the kit. (4) HE staining of the liver.

\section{Statistical analysis}

SPSS17.0 statistical software was used for statistical analysis. The data were measured by $\bar{x} \pm \mathrm{s}$. The mean of multiple groups was compared by one-way ANOVA. Compared with the LSD-t test, the difference between 22 groups was significant.

\section{Results}

The effect of high fructose intake during pregnancy on the weight of young rats

$\mathrm{HF}$ group, SHF group of rats body mass expulsion below the body mass of rats in BC Group $(\mathrm{P}<0.05)$ see table 1 .

\begin{tabular}{|c|c|}
\hline Group & weight ( g ) \\
\hline BC & $5.92 \pm 0.28$ \\
\hline NF & $5.88 \pm 0.41$ \\
\hline HF & $5.63 \pm 0.46^{a}$ \\
\hline SHF & $5.58 \pm 0.25^{\mathrm{a}}$ \\
\hline SHS & $5.59 \pm 0.33$ \\
\hline
\end{tabular}

a means a difference between the control group and the control group

The results of glucose tolerance test in each group Different doses of fructose intake group delivered rats OGTT test in eighth weeks. Results from table 3 and figure 1 showed that the blood glucose absorption reached a peak at about $30 \mathrm{~min}$. At $30 \mathrm{~min}$, the blood glucose values of HF group, SHF group and SHS group were all higher than those of BC group and NF group, with a statistically significant difference $(\mathrm{P}<0.05)$. At $120 \mathrm{~min}$, the blood glucose values of group $\mathrm{HF}$, group
SHF and group SHS were higher than those of BC group $(\mathrm{P}<0.05)$. The blood glucose values of SHF group and SHS group were significantly different from those of NF group ( $\mathrm{P}<0.05)$. In addition, at 120min, the blood glucose values of group $\mathrm{BC}$ and $\mathrm{NF}$ were basically recovered to the initial blood sugar, while the blood sugar values in group HF, SHF and SHS were always higher than those of the initial value.

Table 2. Glucose tolerance results in each group ( $\mathrm{mmol} / \mathrm{L}, \overline{\mathrm{X}} \pm \mathrm{s})$

\begin{tabular}{|c|c|c|c|c|}
\hline 组别 & Omin & 30min & 60min & $120 \mathrm{~min}$ \\
\hline$\overline{B C}$ & $3.97 \pm 0.27$ & $4.93 \pm 0.25$ & $4.62 \pm 0.38$ & $3.99 \pm 0.20$ \\
\hline NF & $4.02 \pm 0.22$ & $5.08 \pm 0.66$ & $5.09 \pm 0.57$ & $4.10 \pm 0.23$ \\
\hline HF & $4.10 \pm 0.42$ & $6.03 \pm 0.79^{a b}$ & $5.24 \pm 0.60$ & $4.38 \pm 0.23^{a}$ \\
\hline SHF & $4.29 \pm 0.29^{a}$ & $6.68 \pm 0.58^{a b}$ & $5.26 \pm 0.69$ & $4.41 \pm 0.22^{a b}$ \\
\hline SHS & $4.42 \pm 0.27^{\mathrm{a}}$ & $6.98 \pm 0.85^{\mathrm{ab}}$ & $5.70 \pm 0.61$ & $4.60 \pm 0.38^{a b}$ \\
\hline
\end{tabular}

a means a difference between the control group and the control group , bmeans a difference between the normal group 
Effects of fructose intake at different doses in pregnancy on the level of insulin and the degree of IR in the offspring

The value of fasting blood glucose in group SHF and group SHS was significantly higher than that in group $\mathrm{BC}$, and the difference was statistically significant $(\mathrm{P}<$ $0.05)$. The serum insulin content in group $\mathrm{HF}$, SHF group and SHS group was significantly higher than that of group $\mathrm{BC}$ and $\mathrm{NF}$ group, the difference was statistically significant $(\mathrm{P}<0.05)$. The value of HOMA-IR in group HF, group SHF and group SHS was significantly higher than that of group $\mathrm{BC}$ and NF, and the difference was statistically significant $(\mathrm{P}<$ $0.05)$.

Table 3 . Effects of different doses of fructose intake on the level of insulin and the degree of IR in the

\begin{tabular}{|c|c|c|c|}
\hline 组别 & FBG ( mmol/L ) & FINS ( uIU/ml ) & HOMA-IR \\
\hline BC & $3.97 \pm 0.27$ & $8.78 \pm 0.87$ & $1.59 \pm 0.15$ \\
\hline NF & $4.01 \pm 0.24$ & $10.36 \pm 0.60$ & $1.86 \pm 0.16$ \\
\hline HF & $4.10 \pm 0.42$ & $12.65 \pm 0.83^{\mathrm{ab}}$ & $2.30 \pm 0.24^{\mathrm{ab}}$ \\
\hline SHF & $4.29 \pm 0.29^{\mathrm{a}}$ & $14.75 \pm 0.65^{\mathrm{ab}}$ & $2.81 \pm 0.20^{a b}$ \\
\hline SHS & $4.23 \pm 0.27^{\mathrm{a}}$ & $11.71 \pm 0.53^{a b}$ & $2.30 \pm 0.16^{\mathrm{ab}}$ \\
\hline SHS & $4.42 \pm 0.27^{\mathrm{a}}$ & $5.70 \pm 0$. & $4.60 \pm 0.38^{a b}$ \\
\hline
\end{tabular}

a means a difference between the control group and the control group , bmeans a difference between the normal group

The effect of fructose intake at different doses in pregnancy on the liver of the offspring

The liver cells in the control group and normal fructose group were arranged orderly, while the liver cells in the high dose fructose group and the super high fructose group were disorder, and a large number of fat cells appeared. The fat accumulation in the high dose fructose group was more serious.
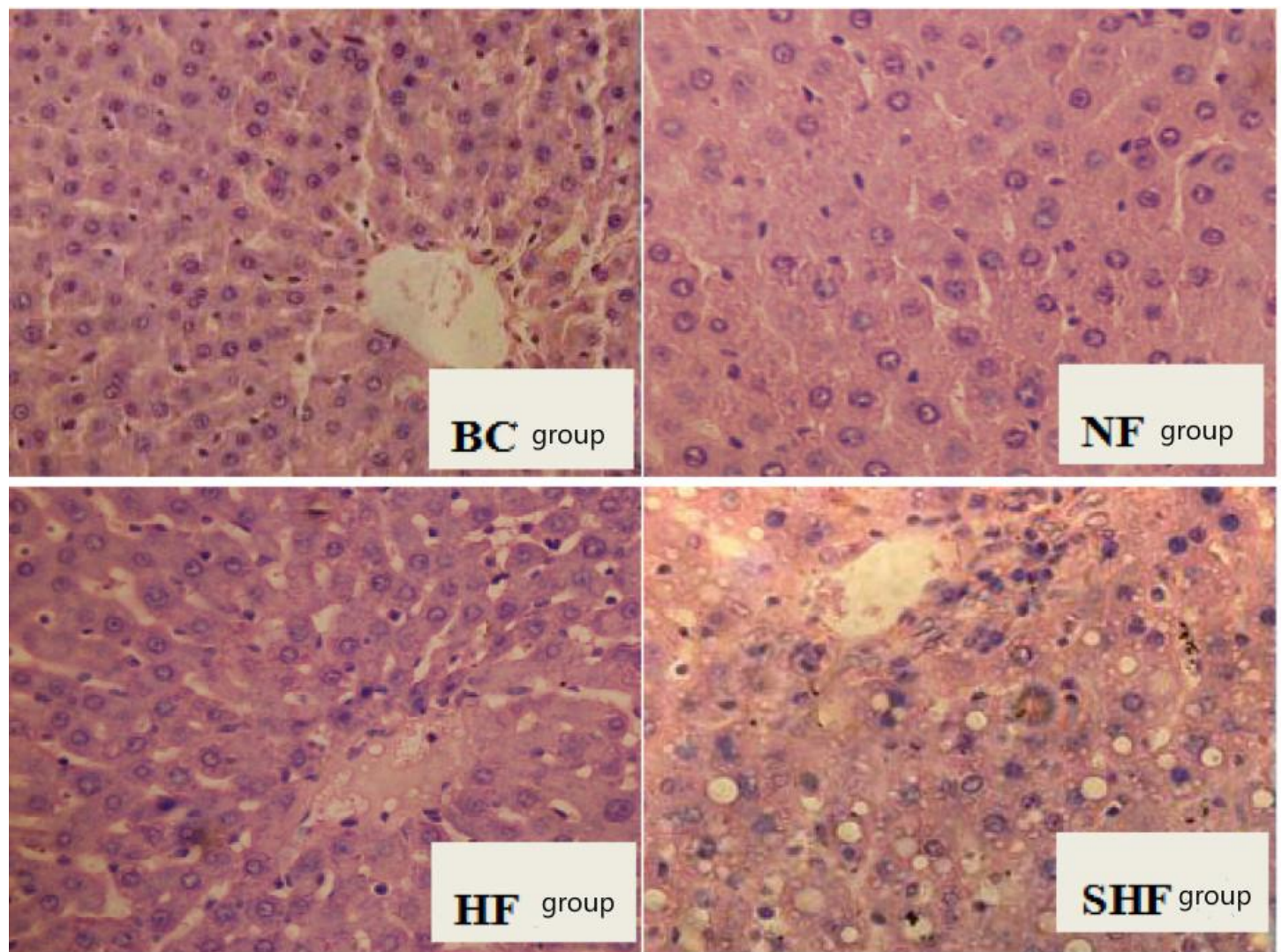

Fig.1 Pathological section of liver HE staining in each group of offspring rats ( x100 )

\section{Discussion}

This research shows that: the pregnancy high fructose intake offspring of FBS and FINS were increased, the difference was statistically significant $(\mathrm{P}<0.05)$, indicating that the pregnancy high fructose diet can cause sub sugar metabolic abnormalities, the research results are consistent with previous ${ }^{[3]}$. HF group, SHF group of rats body mass expulsion below the body mass of BC rats in Group VIII, the difference was statistically significant $(\mathrm{P}<0.05)$. This is consistent with the conclusions of Seghieri $\left[{ }^{4]}\right.$ et al. Intrauterine adverse environment is easy to cause low birth weight in offspring, while low birth weight increases the risk of offspring insulin resistance. Sub sugar metabolic 
abnormalities may increase the risk of many chronic diseases, the study showed that ${ }^{[5]}$ during pregnancy: excessive intake of fructose, can cause intrauterine environment changes, it will stimulate the damaged islet cells of newborn rats offspring after birth, after a period of pancreatic apoptosis and recombination, although in adult offspring the number of islets has been restored, but there is insufficient secretion and insulin resistance. This is in agreement with the conclusions obtained in this study.

From a histological point of view, nonalcoholic fatty liver diseases include simple nonalcoholic steatohepatitis, liver inflammation, and liver fibrosis . Nonalcoholic fatty liver disease and simple nonalcoholic hepatitis are all caused by obesity and insulin resistance, which increase the level of free fatty acids and increase the inflammatory effect by. Up to $30 \%$ of non - alcoholic fatty liver disease patients have the risk of progression of inflammatory infiltration and fibrosis, which ultimately leads to of fatty hepatitis. Most of the patients were examined in the laboratory for , and the indexes of the liver examination were normal. It can be seen from the results that the actual prevalence of non-alcoholic fatty liver disease should be higher than that of the present. More and more evidence that nonalcoholic fatty liver disease is a risk factor for cardiovascular disease, nonalcoholic fatty liver disease will accelerate the occurrence and development of for cardiovascular disease, nonalcoholic fatty liver disease can lead to endothelial dysfunction, thereby accelerating the development of early atherosclerosis, so that the prevalence of cardiovascular disease the rate of increase of.

According to the theory of fetal origin, if there is a hazard in the important stage of embryo development, the growth of tissue or cell will change to make up for the damage, that is, biological programming. During pregnancy, malnutrition and metabolic disorders have been confirmed with the growth and development of offspring is closely related to research also pointed out: in the stage of pregnancy is not a reasonable diet will make the adult offspring of risk after the occurrence of insulin resistance and hypertension, diabetes, coronary heart disease, stroke and other diseases increased significantly. Now more and more people because of early life adverse environmental changes and long-term effects caused by its use of "programming" theory to elaborate, therefore, on the basis of the "programming" theory can be explained in the adult stage of chronic non communicable diseases: diabetes, hypertension, dyslipidemia and insulin resistance based metabolic syndrome with early life adverse intrauterine environment, because of the adverse factors of adverse intrauterine environment stimulates adaptive adjustment or permanent change of fetal ${ }^{[6] . ~ T h i s ~ a l s o ~}$ explains to some extent the cause of the rapid increase in the incidence of insulin resistance and metabolic syndrome. The results of this study showed that excessive intake of fructose during pregnancy increased the incidence of ${ }^{[7]}$ in the adult IR after adulthood.

\section{Conclusion}

This experiment showed gestational high fructose intake on glucose metabolism of offspring had a serious impact, so as to further expose the harm of high fructose diet on offspring caused by the crowd for reasonable and safe daily intake of fructose to provide a certain reference value, especially to the correct guidance of pregnancy high fructose diet, a reasonable intake of fructose, thus reducing the risk of offspring adverse outcome.

\section{References}

1. Malcolm J. Through the looking glass: gestational diabetes as a predictor of maternal and offspring long-term health[J]. Diabetes, 2012, 28(4):307-311.

2. Kajantie E, Eriksson J, Osmond C, et al. Size at birth, the metabolic syndrome and 24-h salivary cortisol profile[J]. Clinical Endocrinology, 2004, 60(2):201-207.

3. MALCOLM J. Through the looking glass: gestational diabetes as a predictor of maternal and offspring long-term health [J]. Diabetes Metab Res Rev, 2012, 28(4): 307-11.

4. PETRY C J, DORLING M W, PAWLAK D B, et al. Diabetes in old male offspring of rat dams fed a reduced protein diet $[\mathrm{J}]$. Int J Exp Diabetes Res, 2001, 2(2): 139.

5. SCAGLIA L, CAHILL C J, FINEGOOD D T, et al. Apoptosis participates in the remodeling of the endocrine pancreas in the neonatal rat [J]. Endocrinology, 1997, 138(4): 1736-41.

6. NATHANIELSZ P W, POSTON L, TAYLOR P D. In utero exposure to maternal obesity and diabetes: animal models that identify and characterize implications for future health [J]. Obstetrics \& Gynecology Clinics of North America, 2007, 34(2): 201-12.

7. SYMONDS M E, PEARCE S, BISPHAM J, et al. Timing of nutrient restriction and programming of fetal adipose tissue development [J]. Proc Nutr Soc, 2004, 63(3): 397-403 